\title{
HOLOMORPHICITY OF A CLASS OF SEMIGROUPS OF MEASURES OPERATING ON $L^{p}(G / H)$
}

\author{
TOMASZ PRZEBINDA
}

\begin{abstract}
In the present paper we consider the class of stable semigroups of measures on a Lie group $G$. This class contains the Gaussian semigroups. We prove that under certain strongly continuous representations of $G$ acting in $L^{\prime \prime}(G / H)$. $1 \leqslant p<x$. these semigroups are holomorphic and uniformly bounded
\end{abstract}

Introduction. For a fixed Lie group $G$. let $(S)$ denote the smallest family of semigroups of measures in $G$ that contains all Gaussian semigroups (i.e., those semigroups whose infinitesimal generators are sub-Laplacians) and is closed with respect to taking sums of generators and subordination. Hulanicki [2] has posed the problem of determining if semigroups in $(S)$ are holomorphic. It is known that Gaussian semigroups are holomorphic [5], but beyond this, additional assumptions are needed. For example, if $G$ is a class two nilpotent group, any semigroup in $(S)$ with $L^{2}$ densities is holomorphic [3]. In this paper we consider semigroups in the image of $(S)$ under strongly continuous representations of $G$, and show that. for a certain class of representations, these semigroups are holomorphic.

Preliminaries. We identify the Lie algebra of $G$, ạ, with left-invariant differential operators by setting

$$
X f(x)=\left.\frac{d}{d t} f(\exp t X)\right|_{t=0} .
$$

For fixed basis $\left\{X_{1}, \ldots, X_{n}\right\}$ of $g$ and multi-index $z=\left(z_{1}, \ldots, z_{n}\right)$ we set $|\mathbf{z}|=z_{1}$ $+\cdots+z_{n}$ and

$$
X^{z} f=X_{i}^{i} \cdots X_{n}^{i n} f .
$$

We denote by $C_{0}(G)$ the space of continuous real-valued functions on $G$ that vanish at infinity, and for a positive integer $k$ we set

$$
C_{0}^{h}=\left\{f \in C_{0}(G) \mid X^{\prime} f \in C_{0} \text { for }|\mathbf{z}| \leqslant k\right\} .
$$

$C_{0}(G)$ is given the topology of uniform convergence, and $C_{1}^{h}(G)$, the topology of uniform convergence for derivatives of order $k$.

Received by the editors October 12. 1981 and, in revised form. May 27. 1982

1980 Mathematics Subject Classification. Primary 47D()5: Secondary 43A10. 22D15. 22E45

Kel nords and phrases. Semigroups of measures, holomorphic semigroups, Lic groups. Haar measure, absolutely continuous measures, group representations. 
We identify $C_{0}(G)^{*}$ with $M(G)$. the space of bounded. regular Borel measures on $G . M(G)$ is a Banach *-algebra with respect to convolution $\mu * \nu$. defined by

$$
\langle\mu * \nu, F\rangle=\int_{G_{i}} \int_{G_{i}} F(x y) d \mu(x) d \nu(y) .
$$

and involution $\mu^{*}$, defined by

$$
\left\langle\mu^{*}, F\right\rangle=\int_{G} F\left(x^{-1}\right) d \mu(x) .
$$

We let $P(G)$ denote the subalgebra of nonnegative measures with norm $\leqslant 1$. A subset $\left\{\mu_{t}\right\}_{t, 0} \subseteq P(G)$ is said to be a semigroup of measures on $G$ if $\mu_{s} * \mu_{t}=\mu_{1+1}$ and

$$
\lim _{t \rightarrow 0}\left\|\mu_{t} * f-f\right\|_{x}=0, \quad f \in C_{0}(G) .
$$

We denote by $C^{\infty}(G)$ the space of test functions on $G$, and by ${ }^{\infty}(G)$ the space of distributions on $G$. A distribution $D$ is said to be dissipative if $\langle D, f\rangle \leqslant 0$ whenever $f \in C_{r}^{\infty}(G)$ and $f(e)=\|f\|_{x}$.

Given a semigroup of measures on $G,\left\{\mu_{t}\right\}_{t},(0)$. the infinitesimal generator of $\left\{\mu_{t}\right\}_{t},(), A$, is the closed operator on $C_{0}(G)$ defined by

$$
A f=\lim _{t: 0} \frac{1}{t}\left(\mu_{t} * f-f\right) \text {. }
$$

where the domain $\mathrm{D}(A)$ of $A$ is the subset of $C_{0}(G)$ for which the right-hand side exists. One has that $C_{c}^{x}(G) \subseteq \mathrm{D}(A)$ and that the mapping $f \rightarrow A f(e)$ is a dissipative distribution on $G$. Conversely, given a dissipative distribution $D$ on $G$, define the operator $A_{D}$ on $C_{c}^{x}(G)$ by $A_{D} f(x)=\left\langle D, f_{x}\right\rangle$. Then $A_{D}$ is closable in $C_{0}(G)$ and is the infinitesimal generator of a unique semigroup of measures on $G$. (These results are essentially due to Hunt [4], with more modern treatments found in [1 and 2].)

Let $\left\{\mu_{t}\right\}_{t}>0$ and $\left\{\nu_{t}\right\}_{t}>0$ be semigroups of measures on $G$ with infinitesimal generators $A$ and $B$, respectively. Clearly, $f \rightarrow(A+B) f(e)$ is a dissipative distribution. and hence there is a unique semigroup whose infinitesimal generator on $C_{c}^{x}(G)$ is $A+B$. Also, given $0<a<1$, the distribution

$$
f \rightarrow-\Gamma(-a)^{-1} \int_{0}^{x} t^{-a-1}\left(\mu_{t} * f-f\right)(e) d t
$$

is dissipative. We denote the corresponding semigroup of measures by $\left\{\mu_{t}^{(a)}\right\}_{t>0}$ and its infinitesimal generator by $|A|^{a}$. $\left\{\mu_{t}^{(a)}\right\}_{t>0}$ is said to be subordinate to $\left\{\mu_{t}\right\}_{t}>0$.

Given a subset $\left\{X_{1} \ldots, X_{k}\right\} \subseteq \mathrm{a} . f \rightarrow\left(X_{1}^{2}+\cdots+X_{k}^{2}\right) f(e)$ is a dissipative distribution. Such distributions generate the Gaussian semigroups. The family of stable semigroups, $(S)$, consists of those semigroups of measures whose corresponding dissipative distribution belongs to $S(\mathrm{~g})=\cup_{k=0}^{x} S_{k}(\mathrm{~g})$, where

$$
\begin{aligned}
S_{0}(\mathfrak{g}) & =\left\{f \rightarrow X^{2} f(e) \mid X \in \mathfrak{a}\right\}, \\
S_{2 h}(\mathfrak{a}) & =\left\{D_{1}+D_{2} \mid D_{1} \in S_{2 k-1}(\mathfrak{a})\right\} \cup S_{2 h-1}(\mathfrak{a})
\end{aligned}
$$


and

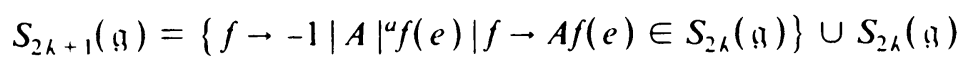

for $k=1,2, \ldots$ For $D \in S(a)$, the hull of $D$ is the smallest Lie subalgebra of $!, b$, such that $D \in S(\mathfrak{b})$.

\section{Holomorphicity of represented semigroups.}

THEOREM 1. Let $\left\{\mu_{t}\right\}_{t>0}$ be a stable semigroup of measures on $G$ whose corresponding dissipative distribution has hull a as above. There exists a $0<\theta \leqslant \pi / 2$, a positive integer $p$, and a family of holomorphic functions $\left\{\omega_{\mathbf{n}} \mid \mathbf{n} \in\left(\mathbf{Z}^{+}\right)^{p}\right\}$ defined on $\Omega_{\theta}=$ $\{Z \in \mathbf{C}|| \arg Z \mid<\boldsymbol{\theta}\}$ with values in $M(G)$ satisfying

(i) $\omega_{n}(t) \geqslant 0$ and $\left\|\omega_{n}(t)\right\|=1$, for $t>0$.

(ii) $\lim _{n_{1}, x} \cdots \lim _{n_{n}, x} \omega_{n}(t) * f=\mu_{t} * f$ for $t>0 . f \in L^{\prime}(G)$,

(iii) there is an $n_{0} \in \mathbf{Z}^{+}$such that for $n_{1} \geqslant n_{0}, 1 \leqslant i \leqslant p$, and $z \in \Omega_{\theta}, \omega_{\left(n_{1} \ldots, n_{n},\right)}(z)$ $\in L^{\prime}(G)$.

Proof. The proof is by induction on the smallest integer $k$ such that dissipative distribution $D$ corresponding to $\left\{\mu_{t}\right\}_{t>0}$ is in $S_{k}(a)$. If $k=0$ then $\operatorname{dim}(\eta)=1$ and the theorem follows from well-known facts about the Gaussian semigroup on $\mathbf{R}$ or $\mathbf{T}$. For example, on $\mathbf{R}$ we take $\boldsymbol{\theta}=\pi / 2, p=1$ and for $z \in \Omega_{\theta}, n \in \mathbf{Z}^{+}$, we have $\omega_{n}(z)=\omega_{1}(z)=(4 \pi z)^{1 / 2} e^{-x^{2} / 4 z} d x$, where $d x$ is Lebesgue measure on $\mathbf{R}$.

We assume the theorem for $j<2 k+1$ and let $D \in S_{2 k+1}(q)$. There is a $D_{1} \in$ $S_{2 h}(\mathfrak{g})$ and a $0<a<1$ so that $\mu_{t}=\nu_{t}^{(a)}$ where $\left\{\nu_{t}\right\}_{t, 0)}$ is generated by $D_{1}$. Now, the hull of $D_{1}$ is $\underline{a}$, and hence, by induction there exist, $\theta_{1}, p_{1}$ and $\left\{\omega_{n}^{1} \mid \mathbf{n} \in\left(\mathbf{Z}^{+}\right)^{p_{1}}\right\}$ satisfying the conditions of the theorem. We let $\theta=a \theta_{1}, p=p_{1}$, and for $z \in \Omega_{\theta}$. $\mathbf{n} \in\left(\mathbf{Z}^{+}\right)^{p}$, we define

$$
\omega_{n}(z)=\int_{0}^{x} f_{a}(\lambda) \omega_{n}^{1}\left(\lambda z^{1 / a}\right) d \lambda
$$

where

$$
f_{a}(\lambda)=\frac{1}{2 \pi i} \int_{\sigma-1 x}^{\sigma+1 x} e^{i \lambda-z^{u}} d z, \quad \sigma>0, \lambda \geqslant 0 .
$$

Conditions (i) and (ii) then follow immediately from the properties of $f_{a}$ and for (ii) we have, for $t>0$ and $f \in L^{\prime}(G)$,

$$
\begin{aligned}
\lim _{n_{1} \rightarrow \infty} \cdots \lim _{n_{p} \rightarrow \infty} \omega_{n}(t) * f & =\lim _{n_{1} \rightarrow \infty} \cdots \lim _{n_{p} \rightarrow \infty} \int_{0}^{x} f_{a}(\lambda) \omega_{n}^{1}\left(\lambda t^{1 ; a}\right) * f d \lambda \\
& =\int_{0}^{\infty} f_{a}(\lambda) \nu_{\lambda t^{1 ; a}} * f d \lambda=\lambda_{t}^{(a)} * f .
\end{aligned}
$$

(The reader is referred to [8], for properties of $f_{a}$.)

Finally, we assume the theorem for $j<2 k$ and let $D \in S_{2 k}(g)$. Let $D_{1}, D_{2} \in$ $S_{2 k-1}(g)$ such that $D=D_{1}+D_{2}$. Let $g_{i}$ be the hull of $D_{1}$ and let $G_{1}$ be the 
corresponding connected Lie subgroups of $G$. (Note that $a$ is generated by $a_{1}$ and (? $_{2}$.) There exist $\theta_{1}, p_{l}$, and $\omega_{\mathbf{n}}^{l}$ for $\mathbf{n} \in\left(\mathbf{Z}^{+}\right)^{p}$, satisfying the conditions of the theorem with respect to $G_{i}$. Let $\theta=\min \left\{\boldsymbol{\theta}_{1}, \boldsymbol{\theta}_{2}\right\}, p=p_{1}+p_{2}+1$, and for $z \in \Omega_{\theta}, \mathbf{n} \in\left(\mathbf{Z}^{+}\right)^{p}$ let

$$
\omega_{n}(z)=\left(\omega_{n^{1}}^{1}\left(z / n_{p}\right) * \omega_{n}^{2} z\left(z / n_{p}\right)\right)^{n},
$$

where $\mathbf{n}=\left(\mathbf{n}^{1}, \mathbf{n}^{2}, n_{p}\right) \cdot \omega_{\mathbf{n}}$ is holomorphic on $\Omega_{\theta}$ and satisfies (i). Furthermore. if $\left\{\mu_{t}^{\prime}\right\}_{1>0}$ is the semigroup generated by $D_{i}$, then, for $f \in L^{\prime}(G), t>0$. one has, by Chernoff's theorem, that

$$
\begin{aligned}
\lim _{n_{1} \rightarrow \infty} \cdots \lim _{n_{p} \rightarrow \infty} \omega_{n}(t) * f & =\lim _{n_{1} \rightarrow \infty} \cdots \lim _{n_{p} \rightarrow \infty}\left(\omega_{n^{\prime}}^{1}\left(t / n_{p}\right) * \omega_{n^{2}}^{2}\left(t / n_{p}\right)^{n_{p}} * f\right. \\
& =\lim _{n_{p} \rightarrow \infty}\left(\mu_{t / n_{p}}^{1} * \mu_{t / n_{p}}^{2}\right)^{n_{p}} * f=\mu_{t} * f .
\end{aligned}
$$

Condition (iii) is an immediate consequence of a known theorem: ${ }^{1}$ Let $G_{1} \ldots, G_{h}$ be connected Lie subgroups of the Lie group $G$ whose Lie algebras generate the Lie algebra of $G$. There is an integer $N$ such that

$$
\left(L^{\prime}\left(G_{1}\right) * L^{\prime}\left(G_{2}\right) * \cdots * L^{\prime}\left(G_{k}\right)\right)^{N} \subseteq L^{\prime}(G) .
$$

Let $H$ be a closed subgroup of the connected Lie group $G$ and suppose there is a measure on $G / H$ that is invariant with respect to the action of $G$. Suppose further that $\gamma: G \times G / H \rightarrow \mathbf{C}$ is a continuous function with $\|\gamma\|_{\infty} \leqslant 1$ such that, for $1 \leqslant p<\infty$, the mapping $y \rightarrow \Gamma_{y}^{p}$ of $G$ into the bounded operators on $L^{p}(G / H)$ given by

$$
\Gamma_{y}^{p} F(\dot{x})=\gamma(y, \dot{x}) F\left(y^{-1} \dot{x}\right)
$$

for $F \in L^{p}(G / H)$ and a.e. $\dot{x} \in G / H$ defines a strongly continuous representation of $G$ that is unitary for $p=2$.

TheOREM 2. Let $G$ be a connected Lie group, let $\left\{\mu_{t}\right\}_{t>0} \in(S)$, and suppose that the hull of the distribution generating $\left\{\mu_{t}\right\}_{t>0}$ is the Lie algebra of $G$. Let $H$ and $\gamma$ be as above and suppose that $\Gamma^{p}\left(L^{1}(G)\right)$ is contained in the space of compact operators on $L^{p}(G / H)$ for $1 \leqslant p<\infty$. There is a $0<\theta \leqslant \pi / 2$ and a holomorphic $T^{p}: \Omega_{\theta} \rightarrow$ $B\left(L^{p}(G / H)\right)$ such that

(i) $\sup \left\{\left\|T_{z}^{p}\right\| \mid z \in \Omega_{\theta}\right\} \leqslant 1$,

(ii) for $t>0, T_{t}^{p}=\Gamma^{p}\left(\mu_{t}\right)$.

Proof. Let $\theta, p$ and $\omega_{n}$ be as in Theorem 1. Notice that $\omega_{1}(t)$ is the semigroup of probabilistic measures generated by $X_{1}^{2}$, whose image $\Gamma^{2}\left(X_{1}^{2}\right)=A_{1}$ is an essentially selfadjoint operator [1, Theorem 12, Example 4] and generates the semigroup of contractions $\Gamma^{2}\left(\omega_{1}(t)\right)$, so that

$$
\Gamma^{2}\left(\omega_{1}(z)\right)=\int_{-\infty}^{0} e^{\lambda z} d P(\lambda)
$$

\footnotetext{
'See remarks.
} 
where $P$ is the spectral measure of $A_{1}$, and hence $\left\|\Gamma^{2}\left(\omega_{1}(z)\right)\right\| \leqslant 1$ for $\operatorname{Re}(z)>0$. Now by an induction argument analogous to that used in the proof of Theorem 1 , one can show that for, $f \in L^{p}(G / H), 1 \leqslant p$ and $t>0$,

$$
\lim _{n_{1} \rightarrow x} \cdots \lim _{n_{p} \rightarrow x} \Gamma^{p}\left(\omega_{n}(t)\right) f=\Gamma^{p}\left(\mu_{t}\right) f
$$

and that $\left\|\Gamma^{2}\left(\omega_{n}(z)\right)\right\| \leqslant 1$ for $z \in \Omega_{\theta}$.

Let $n_{0}$ be as in Theorem 1, and set $\mathbf{Z}_{0}=\left\{k \in \mathbf{Z}, k>n_{0}\right\}$. Then if $\mathbf{n} \in\left(\mathbf{Z}_{0}\right)^{n}$. $\omega_{n}(z) \in L^{\prime}(G)$ for $z \in \Omega_{\theta}$, and so $\Gamma^{p}\left(\omega_{n}(z)\right)$ and $\Gamma^{2}\left(\omega_{n}(z)\right)$ are compact operators. By [6], their spectra coincide, and hence also their norms. Thus $\left\|\Gamma^{\rho}\left(\omega_{n}(z)\right)\right\| \leqslant 1$. Therefore. $\left\{\Gamma^{p}\left(\omega_{\mathbf{n}}\right) \mid \mathbf{n} \in\left(\mathbf{Z}_{0}\right)^{p}\right\}$ is a family of holomorphic functions on $\Omega_{\theta}$ that is uniformly bounded and convergent on $\mathbf{R}^{+}$. Thus, by Vitali's theorem.

$$
\lim _{n_{1} \rightarrow x} \cdots \lim _{n_{p} \rightarrow x} \Gamma^{p}\left(\omega_{n}(z)\right)=T_{z}^{p}
$$

exists for $z \in \Omega_{\theta}$. and $z \rightarrow T_{z}^{p}$ is holomorphic.

Remarks. 1. I have learned from Joe W. Jenkins and Andrzej Hulanicki that the theorem mentioned at the end of the proof of Theorem 1 was known but I could not find it in the literature. Here is a rough outline of a proof.

Assume for simplicity that $k=2$. Using the fact that the zero set of a nontrivial real analytic function defined on an open subset of $\mathbf{R}^{\prime \prime}$ has Lebesgue measure zero. one can prove the following:

Lemma. Let $M$ and $G$ be tw'o real analytic manifolds of dimensions $N$ and $n$ respectively $(N \geqslant n)$, with Lebesgue measures $\mu$ and $\nu$. Suppose that $M$ is connected. If $F: M \rightarrow G$ is a real analytic function whose derivative has rank $n$ at a point in $M$. then $F(\mu)$ is absolutely continuous with respect to $\nu$.

Let $a_{1}$, be the Lie algebra of $G$, with basis $\left\{X_{11}, X_{12}, \ldots, X_{t p}\right\}(i=1,2)$. Let

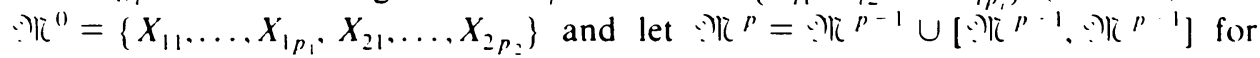
$p=1.2 .3 \ldots$ The assumption that $\natural_{1} \cup \mathrm{g}_{2}$ generates $a$ implies that there is such a $p$ that $\because r^{p}$ contains the basis $\left\{X_{1}, X_{2}, \ldots, X_{n}\right\}$ of $q$. The Baker-CampbellHausdorff formula [7. Theorem 2.15.4] implies that there exists an $m$ such that for every $X \in)^{n}$ there is a function $S_{X}: \mathbf{R} \rightarrow \mathbf{R}^{m\left(p_{1}+p_{2}\right)}$ such that for $|t|$ small enough we have $f_{m}\left(S_{X}(t)\right)=\exp \left(t X+r_{X}(t)\right)$, where

$$
\begin{aligned}
& f_{m}\left(u_{111}, u_{112}, \ldots, u_{11 p_{1}}, u_{121}, u_{122}, \ldots u_{12 p_{2}}, \ldots, u_{m 11}\right. \text {. } \\
& \left.u_{m 12}, \ldots, u_{m 1 p_{1}}, u_{m 21}, u_{m 22}, \ldots, u_{m 2 n_{2}}\right) \\
& =\exp \left(\sum_{1=1}^{p_{1}} u_{11}, X_{1}\right) \exp \left(\sum_{j=1}^{p_{2}} u_{12,} X_{2,}\right) \cdots \exp \left(\sum_{,=1}^{p_{1}} u_{m 1}, X_{1}\right) \exp \left(\sum_{i=1}^{p_{2}} u_{m 2,}, X_{2,}\right)
\end{aligned}
$$

and

$$
r_{X}(t)=\sum_{\alpha+\beta>2 n^{\prime}}|t|^{(\alpha+\beta) / 2 n}(\operatorname{sign}(t))^{\varepsilon_{n+1}} X_{\alpha \beta} \quad\left(X_{\alpha \beta} \in \underline{a}, \varepsilon_{\alpha \beta} \in\{0,1\}\right) .
$$


For example, if $p_{1}=p_{2}=1$ and $X=\left[X_{11}, X_{21}\right]$ then $m=2$ and

$$
S_{X}(t)=\left(|t|^{1 / 2},|t|^{1 / 2} \operatorname{sign}(t),-|t|^{1 / 2},-|t|^{1 / 2} \operatorname{sign}(t)\right) \text {. }
$$

Choose $S_{x_{1}}$ as above for every vector $X_{i}$ from the basis of $\mathfrak{g}$, and put $S(\mathbf{t})=$ $S\left(t_{1}, t_{2}, \ldots, t_{n}\right)=\left(S_{X_{1}}\left(t_{1}\right), S_{X_{2}}\left(t_{2}\right), \ldots, S_{X_{n}}\left(t_{n}\right)\right) \in \mathbf{R}^{N\left(p_{1}+P_{2}\right)}$ where $N=n m$. Define $M=G_{1} \times G_{2} \times \cdots \times G_{1} \times G_{2}(N$ times $)$.

$M \ni\left(x_{11}, x_{12}, \ldots, x_{N 1}, x_{N 2}\right) \rightarrow F\left(x_{11}, x_{12}, \ldots, x_{N 1}, x_{N 2}\right)=x_{11} x_{12} \cdots x_{N 1} x_{N 2} \in G$ and $\Psi: \mathbf{R}^{N\left(p_{1}+p_{2}\right)} \rightarrow M$ by the formula

$$
\begin{aligned}
\Psi\left(u_{111}, u_{112}, \ldots, u_{11 p_{1}}, u_{121}, u_{122}, \ldots, u_{12 p_{2}}, \ldots, u_{N 11},\right. \\
\left.u_{N 12}, \ldots, u_{N 1 p_{1}}, u_{N 21}, u_{N 22} \ldots \ldots u_{N 2 p_{2}}\right) \\
=\left(\exp \left(\sum_{i=1}^{p_{1}} u_{11}, X_{1}\right), \exp \left(\sum_{j=1}^{p_{2}} u_{12}, X_{2}, \ldots, \ldots\right.\right. \\
\left.\quad \exp \left(\sum_{i=1}^{p_{1}} u_{N 1,} X_{1 i}\right), \exp \left(\sum_{,=1}^{p_{2}} u_{N 2}, X_{2}\right)\right) .
\end{aligned}
$$

Thus

$$
\begin{aligned}
F \circ \Psi \circ S(\mathbf{t}) & =f_{m}\left(S_{X_{1}}\left(t_{1}\right)\right) f_{m}\left(S_{X_{2}}\left(t_{2}\right)\right) \cdots f_{m}\left(S_{X_{n}}\left(t_{n}\right)\right) \\
& =\exp \left(t_{1} X_{1}+r_{X_{1}}\left(t_{1}\right)\right) \exp \left(t_{2} X_{2}+r_{X_{2}}\left(t_{2}\right)\right) \cdots \exp \left(t_{n} X_{n}+r_{X_{n}}\left(t_{n}\right)\right)
\end{aligned}
$$

in a neighborhood of the origin in $\mathbf{R}^{n}$, which implies that the Jacobian $J$ of the map $F \circ \Psi \circ S$ is equal to the identity at $t=0$ and that there exists a neighborhood $U$ of the origin in $\mathbf{R}^{n}$ such that for $\mathbf{t} \in U, J(\mathbf{t}) \neq 0$. Take $\mathbf{t}$ from $U$ such that $t_{1} \neq 0$ for each $i=1,2,3, \ldots, n$. Then

$$
\begin{aligned}
n & =\operatorname{rank}\left(T_{1}(F \circ \Psi \circ S)\right)=\operatorname{rank}\left(T(F)_{\Psi(S(t))} \circ T_{1}(\Psi \circ S)\right) \\
& \leqslant \operatorname{rank}\left(T(F)_{\psi(S(t))}\right) \leqslant n .
\end{aligned}
$$

so $\operatorname{rank}\left(T(F)_{\psi_{(S(t))}}\right)=n$. By the lemma $F\left(\mu_{1} \times \mu_{2} \times \cdots \times \mu_{1} \times \mu_{2}\right)$ is absolutely continuous with respect to the Haar measure $\nu$ on $G$, i.e.

$$
\left(\mu_{1} * \mu_{2}\right)^{N}(E)=\mu_{1} \times \mu_{2} \times \cdots \times \mu_{1} \times \mu_{2}\left(F^{-1}(E)\right)=0
$$

for every set $E$ with $\nu(E)=0$.

2. Theorem 2 gives only a partial answer to Hulanicki's question: determine if semigroups in $(S)$ are holomorphic. The proof and the boundedness of $\left\|T_{z}^{p}\right\|$ for $z \in \Omega_{\theta}$ depend very much on the assumption that those operators are compact. Suppose $G$ is a locally compact group and $H$ is a subgroup of $G$ such that there exists an invariant measure on $G / H$. Moreover assume that $\gamma=1$ and $G / H$ is not compact. Then one can show [6] that if $f \in L^{\prime}(G)$ and $\int_{c} f(y) d y \neq 0$ it follows that $\Gamma^{p}(f)$ is not compact in any $L^{p}(G / H), 1 \leqslant p<\infty$. The reader who is interested in the importance of having the semigroups holomorphic on all $L^{p}$ spaces, $1 \leqslant p<\infty$. is referred to [2]. 
The author is very grateful to Joe $\mathrm{W}$. Jenkins for many simplifications and corrections in this paper and to Romuald Dabrowski. Pawel Glowacki. Andrzej Hulanicki, and Czelsaw Ryll-Nardzewski for many discussions on the subject.

\section{REFERENCES}

1. M. Duflo. Representations de semi-gronup de measures sur un groupe localement compact. Ann. Inst. Fourier (Grenoble) 28 (1978), 225-249.

2. A. Hulanicki, A class of comeolution semi-groups of measures on a l.ce group. Lecture Notes in Math. Springer-Verlag. Berlin and New York (to appear).

3. private communication.

4. (i. A. Hunt, Semi-groups of measures on Lie groups. Trans. Amer. Math. Soc. 81 (1956), 264-293.

5. J. Kisynski. Holomorphicity of seni-groups of operators generated by sublaplacians on lice groups. Lecture Notes in Math., Springer-Verlag, Berlin and New York.

6. T. Przebinda, Spectrum of convolution operators on $I . "(G / I)$, preprint.

7. V. S. Varadajan, lie groups, lie algehras and their representations. Prentice Hall. Englewood Cliffs. N.J.. 1974

8. K. Yosida. Functional analists, Springer-Verlag, Berlin, (iöttingen and Heidelberg. 1965.

Department of Mathematics, SUNy at Albany, Albany, New York 12222

Department of Mathematics, Technical. University. Wroclaw, Poland

(urrent address: Department of Mathematics. Yale University. Box 2155 Yale Station. New Haven. Connecticut 06520) 